\title{
Computable negativity in two-mode squeezing subject to dissipation
}

\author{
Marcin Dukalski and Yaroslav M. Blanter \\ Kavli Institute of Nanoscience, Delft University of Technology, Lorentzweg 1, 2628 CJ Delft, The Netherlands
}

(Received 8 February 2015; published 24 March 2015)

\begin{abstract}
We study a system of two bosonic fields subject to two-mode squeezing in the presence of dissipation. We find the Lie algebra governing the dynamics of the problem and use the Wei-Norman method to determine the solutions. Using this scheme, we arrive at a closed-form expression for an infinitely dimensional density operator which we use to calculate the degree of entanglement (quantified by Horodeckis' negativity) between the modes. We compare our result to the known continuous variable entanglement measures. We analyze the conditions for entanglement generation and the influence of thermal environments on the state formed. The problem is relevant, in particular, for understanding of quantum dynamics of coupled optical and/or mechanical modes in optomechanical and nanomechanical systems.
\end{abstract}

DOI: 10.1103/PhysRevA.91.033829

PACS number(s): 03.67.Bg, 42.50.Wk, 03.67.Mn, 03.65.Yz

\section{INTRODUCTION}

Entanglement is a fascinating, unique, and classically unparalleled feature of quantum mechanics. In optical systems, entanglement is produced by means of nonlinear media through a process of three- or more- wave mixing, spontaneous down-conversion, or two-mode squeezing [1], where in each case dissipation and the temperature of the environment play a negligible role. Modern quantum optomechanical [2,3] and nanomechanical [4] systems equipped with elements with sufficiently strong nonlinearities could give rise to similar effective squeezing phenomena. Such bosonic systems are subject to dissipation through coupling to the classical environment, which might reduce the degree of produced entanglement. It is therefore very important to get a quantitative understanding of the squeezing versus dissipation interplay, with the aim of producing or maintaining entanglement between bosonic degrees of freedom such as photons (light or microwave quanta) or phonons (vibrational quanta).

The main obstacle in determining the environmentally induced effects on the degree of bosonic entanglement formed is twofold. For one, the Hilbert space of both of the bosonic modes is infinitely dimensional with high- $n$ Fock states $|n\rangle$ contributing to the degree of entanglement formed, and this requires the usage of more advanced entanglement measures. Second, the presence of dissipation requires solving, instead of a Schrödinger equation, a more complicated Lindblad-type master equation which governs the time evolution of the open quantum system [5,6]. The two conditions combined also mean that the solutions to the equations of motion should be determined for an arbitrarily large Hilbert space, requiring us to find the complete infinite set of time-dependent density matrix elements $\rho_{i j, k l}$. The key to determining the solutions, and hence finding the degree of entanglement, will be to recast the problem using the Wei-Norman method (WNM) [7,8]. The WNM amounts to translating from the master equation containing noncommutating (super)operators to a set of new nonlinear differential equations, whose degree of nonlinearity grows with the complexity of the commutator algebra.

In the past, the Wei-Norman method has been applied to study Lie algebra valued problems [9-14], many of which found applications in optical systems. These problems, however, mainly involved Lie algebras spanned by no more than three generators, and if any extensions to the algebras were considered, only their few operator reductions were studied $[15,16]$. Here, we will treat the most general two-modesqueezed problem subject to dissipation involving as many as 15 generators, and we will be able to obtain analytical solutions to a reduced problem spanned by a Lie algebra composed of 10 , and in a special case all 15 generators.

The great advantage of the Wei-Norman method is that it will provide a density operator in a closed form, which we could use to evaluate the generally established entanglement measures, such as negativity [17-19]. We will be able to find an analytical form of negativity stemming from the operator structure alone, and as a result of it one can use it to find the amount of entanglement in the system in a generalized problem involving time-dependent squeeze parameters, non-Markovian baths, or different environments of individual modes, as long as the bosonic operator form remains unchanged. Furthermore, we will show that the explicitly calculated negativity of an infinitely dimensional density operator in some cases is completely compatible with the separability criterion of the continuous variable states [20,21] for this system. Explicit calculation of negativity, though, not only allows one to find whether or not the state is separable, but also how large is the degree of entanglement. Moreover, due to a similarity in the Lie algebra structures, compared to the single-mode-squeezing case, we will see how the solutions show different quantitative and qualitative behavior in two distinct system parameter regimes, underdamped and overdamped ones, and how in a two-mode symmetric system the entanglement measure is the same in both regimes.

This work is structured as follows. In Sec. II, upon introducing the two-mode-squeezing Hamiltonian, we will couple it to a Markovian bath and transfer the Lindblad-type superoperator master equation into a Lie algebra valued problem, where superoperators present in the equations are identified with Lie algebra elements. Further, we present the generic form of the solution of the problem with the system initialized in a vacuum state, which allows us in Sec. III to determine the degree of entanglement present in the system. In Sec. IV, we compare this result and stemming from it separability condition to that obtained from continuous variable separability condition. Afterwards, in Sec. V, we present the explicit solutions to the master equation and interpret the separability condition 
in terms of the bath temperature, proving that regardless of squeezing strength and dissipation rate the state is inseparable at zero temperature. Additionally, we also investigate the effects of the asymmetric modes-baths coupling strengths and deviation from resonance between the two squeezed modes and the driving mode. Finally, in Sec. VI, we study the case of the system initially in the thermal equilibrium with the environment and we point out a finite-time interval of separability in the transient regime and further show that the same conditions as before are obeyed in the steady-state regime. We close with the conclusions.

\section{SYSTEM}

Let us consider a system comprised of three coupled bosonic modes, with their annihilation operators $\hat{a}, \hat{b}, \hat{c}$, where $\hat{c}$ denotes a strong driving mode, and $\hat{a}$ and $\hat{b}$ are the output modes, with their respective frequencies $\omega_{c}, \omega_{a}$, and $\omega_{b}$. In the presence of a dominant $x^{3}$-type coupling, upon a modal decomposition, and in the regime $\omega_{c} \sim \omega_{a}+\omega_{b}$, the cross interaction terms of the type $\hat{c}^{\dagger} \hat{a}^{\dagger} \hat{b}^{\dagger}, \hat{c}^{\dagger} \hat{a}^{\dagger} \hat{b}$, or $\hat{c}^{\dagger} \hat{a} \hat{b}^{\dagger}$ will be suppressed in the rotating wave approximation. ${ }^{1}$ In this case, the system Hamiltonian will take the form

$$
\hat{H}=\omega_{a} \hat{a}^{\dagger} \hat{a}+\omega_{b} \hat{b}^{\dagger} \hat{b}+\omega_{c} \hat{c}^{\dagger} \hat{c}+g\left(\hat{c}^{\dagger} \hat{a} \hat{b}+\hat{c} \hat{a}^{\dagger} \hat{b}^{\dagger}\right)
$$

which in the interaction picture with respect to all modes takes the form

$$
\begin{aligned}
\hat{\mathcal{V}} & =g\left(\hat{c}^{\dagger} \hat{a} \hat{b} e^{i \delta t}+\hat{c} \hat{a}^{\dagger} \hat{b}^{\dagger} e^{-i \delta t}\right) \\
& =g c\left(\hat{a} \hat{b} e^{i(\delta t+\eta)}+\hat{a}^{\dagger} \hat{b}^{\dagger} e^{-i(\delta t+\eta)}\right),
\end{aligned}
$$

where $\delta=\omega_{c}-\omega_{b}-\omega_{a}$, and where in the second step we have assumed that the driving mode $\hat{c}$ is a very large coherent state with amplitude $c$ and phase $\eta$, and thus will be considered to be frozen out. We can further remove the explicit time dependence of this Hamiltonian by yet another unitary transformation $U^{\dagger} \hat{H} U-i U^{\dagger} \partial_{t} U$, where $U=\exp \left[i \delta t\left(\hat{a}^{\dagger} \hat{a}+\hat{b}^{\dagger} \hat{b}\right)\right]$ and redefining $g c e^{-i \eta} \rightarrow \frac{\xi}{4}$ we get

$$
\hat{H}=\delta\left(\hat{a}^{\dagger} \hat{a}+\hat{b}^{\dagger} \hat{b}\right)+\frac{\xi}{4} \hat{a}^{\dagger} \hat{b}^{\dagger}+\frac{\xi^{*}}{4} \hat{a} \hat{b}
$$

with the factor of $\frac{1}{4}$ included in the definition for future convenience. Assuming a standard coupling of every mode to their respective environments, e.g., phonon or a photon continuum, and assuming that the interaction kernel in each case is time local (Markovian approximation), we arrive at a Lindblad-type master equation

$$
\begin{aligned}
\dot{\rho}= & -i[\hat{H}, \rho]+\kappa_{1}\left(n_{1, t h}+1\right) \mathcal{D}_{\hat{a}} \rho+\kappa_{1} n_{1, t h} \mathcal{D}_{\hat{a}^{\dagger}} \rho \\
& +\kappa_{2}\left(n_{2, t h}+1\right) \mathcal{D}_{\hat{b}} \rho+\kappa_{2} n_{2, t h} \mathcal{D}_{\hat{b}^{\dagger}} \rho,
\end{aligned}
$$

where $\mathcal{D}_{\Theta} \rho=\Theta \rho \Theta^{\dagger}-\frac{1}{2}\left\{\Theta^{\dagger} \Theta, \rho\right\}, \kappa_{i}$ is the dissipation rate, and $n_{i, t h}=\left(e^{\omega_{j} / k_{b} T_{i}}-1\right)^{-1}$ is the thermal occupation number

\footnotetext{
${ }^{1}$ Alternatively these can be considered to be energy-violating terms, which are negligible outside of the ultrastrong coupling regime.
}

in the bath at temperature $T_{i}$ of bosonic mode $i=1,2$ (given by operators $\hat{a}$ and $\hat{b}$, respectively).

For the system initially in the vacuum state $|00\rangle$, Eq. (1) has a solution, that can be written in a form

$$
\begin{aligned}
\rho(t) & =\mathcal{N} \exp \left[f_{3} H_{3}+f_{5} H_{5}+f_{9} H_{9}+f_{12} H_{12}\right]|00\rangle\langle 00|, \\
\mathcal{N} & =\left(1-x_{+}\right)\left(1-x_{-}\right), \quad x_{ \pm}=f_{3} \pm \sqrt{f_{5}^{2}+f_{9}^{2}+f_{12}^{2}},
\end{aligned}
$$

where the numerical prefactor marks the trace-normalization condition and where

$$
\begin{aligned}
H_{3} \rho & =\hat{a}^{\dagger} \rho \hat{a}+\hat{b}^{\dagger} \rho \hat{b}, \quad H_{9} \rho=\hat{a}^{\dagger} \rho \hat{a}-\hat{b}^{\dagger} \rho \hat{b}, \\
H_{5} \rho & =i e^{-i \varphi} \hat{a}^{\dagger} \hat{b}^{\dagger} \rho-i e^{i \varphi} \rho \hat{a} \hat{b}, \\
H_{12} \rho & =e^{-i \varphi} \hat{a}^{\dagger} \hat{b}^{\dagger} \rho+e^{i \varphi} \rho \hat{a} \hat{b}
\end{aligned}
$$

are 4 of the 15 elements of the so(4,2) Lie algebra presented and elaborated on in Appendix A1; the remaining 11 generators drop out due to the initial condition choice. Here, $\varphi=\pi-\operatorname{Arg}(\xi)$. The solution (2), despite being written in a compact form, spans the whole of the infinite-dimensional Hilbert space of both modes. Moreover, the exponents of the superoperators should be understood either in terms of Taylor expansions or in terms of matrix exponents of the matrices operating on the product space

$$
\rho=\hat{A}|n\rangle\left\langle m\left|\hat{B} \rightarrow \tilde{\rho}=\hat{A} \otimes \hat{B}^{\dagger}\right| n\right\rangle \otimes|m\rangle .
$$

In this work, we will adapt the Taylor series approach.

Lastly, quite remarkably thanks to this construction one can obtain analytical expressions for moments

$$
\left\langle\hat{a}^{\dagger k} \hat{a}^{l} \hat{b}^{\dagger m} \hat{b}^{n}\right\rangle=\operatorname{Tr}\left[\hat{a}^{\dagger k} \hat{a}^{l} \hat{b}^{\dagger m} \hat{b}^{n} \rho(t)\right]
$$

in terms of functions $f_{i}$ by skillfully differentiating with respect to $f_{3}, f_{5}, f_{9}$, and/or $f_{12}$ and then renormalizing the moment-generating function

$$
\Lambda=\operatorname{Tr}\left(\exp \left[f_{3} H_{3}+f_{5} H_{5}+f_{9} H_{9}+f_{12} H_{12}\right]\right)=\mathcal{N}^{-1},
$$

for example,

$$
\left\langle\hat{a}^{\dagger} \hat{a}\right\rangle=\frac{1}{2} \mathcal{N}\left(\partial_{f_{3}}+\partial_{f_{9}}\right) \mathcal{N}^{-1},
$$

where from the solution we can see that the only nonzero moments must be of the form $\left\langle\hat{a}^{x+y-z} \hat{a}^{\dagger z} \hat{b}^{y} \hat{b}^{\dagger z}\right\rangle$ with $x, y, z \in$ $\mathbb{Z}$ and $x+y \geqslant z$. This automatically implies that the joint power of the moment must be even, and that of the simplest (quadratic) moments the only nonzero ones are $\left\langle\hat{a}^{\dagger} \hat{a}\right\rangle,\left\langle\hat{b}^{\dagger} \hat{b}\right\rangle$, $\langle\hat{a} \hat{b}\rangle$, and $\left\langle\hat{a}^{\dagger} \hat{b}^{\dagger}\right\rangle$. We will need these in the next section in which we will study the entanglement stored in this bosonic system.

The time-dependent and system-parameters-dependent real functions $f_{3}, f_{5}, f_{9}$, and $f_{12}$ are determined using the WeiNorman method and obey a complicated set of first-order nonlinear differential equations presented in Appendix A2. We postpone the discussion about how the solutions are obtained to Sec. V and, first, in Secs. III and IV, we focus on the entanglement measures as the discussion in terms of the functions $f_{i}$ is more transparent. 


\section{ENTANGLEMENT MEASURES}

The solution in Eq. (2) is described by the application of exponents of creation superoperators on a two-mode vacuum state. If we wish to work with an exact solution and not truncate the Taylor expansion of the superoperator exponent, we arrive at an infinitely dimensional density operator $\rho=|\psi\rangle\langle\psi|$ of a potentially entangled state $|\psi\rangle$. In such a case, the finitedimensional entanglement measures [22,23] no longer apply, which is a reason why here we will attempt to use negativity [17-19] which is not limited by the dimensional restrictions. ${ }^{2}$ The result (2) could also be interpreted as a continuous variable state (CVS), where we could use the entanglement measure bounds imposed by the conditions first presented in Refs. [20,21]. Here, we will show that we can calculate the negativity explicitly, which we will later compare to the CVS separability criterion $[20,21]$. Both of these measures in their core rely on the partial-transposition $p T$ operation, given by

$$
(|i j\rangle\langle k l|)^{p T_{1}}=|k j\rangle\left\langle i l\left|\quad(|i j\rangle\langle k l|)^{p T_{2}}=\right| i l\right\rangle\langle k j|,
$$

i.e., the transposition is taken only with respect to the first and second subspaces, respectively, and we define the negativity as a sum of negative eigenvalues of $\rho^{p T}$. For entangled states defined in a $\operatorname{dim} \times \operatorname{dim}$ dimensional Hilbert space, negativity is a monotonously growing function (an entanglement monotone) with a range $\left[0, \frac{1}{2}(\mathrm{dim}-1)\right]$, giving zero for separable states.

Determination of negativity is not an easy task, however, due to the dimensionality dependence of this entanglement monotone and the infinitely dimensional density operator. Here, every application of $H_{3}, H_{5}, H_{9}, H_{12}$ gives rise to a yet larger Hilbert space and the exponent of these operators results in an infinitely long Taylor expansion. Moreover, negativity is based around negative eigenvalues, which need to be determined. Here, we outline the sketch of a proof which is presented in detail in Appendix A3.

Finding the eigenvalues in this setting amounts to finding the roots of a characteristic polynomial of infinite order, i.e., to solving the equation $\operatorname{det}\left[\rho^{p T r}-I \lambda\right]=0$. Using the determinant of a matrix exponent, exponent of a trace relation, we can write

$$
\operatorname{det}\left[\rho^{\mathrm{pTr}}-I \lambda\right]=\operatorname{det}[-\lambda I] \exp \left\{\sum_{j=1}^{\infty}-\frac{\operatorname{Tr}\left[\left(\rho^{\mathrm{pTr}}\right)^{j}\right]}{j \lambda^{j}}\right\},
$$

which, thanks to the property of the form of the solution (2),

$$
\operatorname{Tr}\left[\left(\rho^{\mathrm{p} \operatorname{Tr}}\right)^{j}\right]=\frac{\left(1-x_{+}\right)^{j}\left(1-x_{-}\right)^{j}}{\left(1-x_{+}^{j}\right)\left(1-x_{-}^{j}\right)},
$$

yields

$$
\operatorname{det}\left[\rho^{\mathrm{p} \operatorname{Tr}}-I \lambda\right]=\operatorname{det}[-\lambda I] \prod_{p, q=0}^{\infty}\left(1-\frac{x_{+}^{p} x_{-}^{q} \mathcal{N}}{\lambda}\right),
$$

\footnotetext{
${ }^{2}$ Other than the presence of bound entanglement in systems with dimensions greater that $2 \times 3$. As we will see in the sections to come, bound entanglement is unlikely to be present.
}

where $x_{ \pm}$were defined before, and where the eigenvalues can be directly read out. Since (as we will later show) $f_{3}$ is always positive, the only negative eigenvalues will have the form $x_{+}^{p} x_{-}^{2 q+1}$ provided that $x_{-}<0$. Upon adding all of them up, we obtain the negativity

$$
\begin{aligned}
N & =\left|\mathcal{N} \sum_{p, q=0}^{\infty} x_{+}^{p} x_{-}^{2 q+1}\right|=\frac{x_{-}}{1+x_{-}} \\
& =\operatorname{Max}\left(0, \frac{-f_{3}+\sqrt{f_{5}^{2}+f_{9}^{2}+f_{12}^{2}}}{1+f_{3}-\sqrt{f_{5}^{2}+f_{9}^{2}+f_{12}^{2}}}\right) .
\end{aligned}
$$

This is the main result of this paper.

\section{CONTINUOUS VARIABLE STATES SEPARABILITY CONDITION}

As first simultaneously and independently formulated by $[20,21]$, the continuous variable states separability criterion stems from quadratic relations of the type

$$
\left\langle\left(\Delta \hat{X}_{\vec{d}}\right)^{2}\right\rangle+\left\langle\left(\Delta \hat{X}_{\vec{d}^{\prime}}\right)^{2}\right\rangle \geqslant\left|d_{1} d_{2}^{\prime}-d_{2} d_{1}^{\prime}+d_{3} d_{4}^{\prime}-d_{4} d_{3}^{\prime}\right|,
$$

which is the Heisenberg uncertainty relation obeyed by all states, with $d_{i}$ and $d_{i}^{\prime}$ being components of the real $\vec{d}$ and $\vec{d}^{\prime}$ four-vectors, $\hat{X}_{\vec{v}}=v_{1} \hat{x}_{1}+v_{2} \hat{p}_{1}+v_{3} \hat{x}_{2}+v_{4} \hat{p}_{2}$, and $\Delta \hat{A}=$ $\hat{A}-\langle\hat{A}\rangle$. Separable states, on the other hand, need to obey a more stricter inequality

$$
\left(\Delta \hat{X}_{\vec{d}}\right)^{2}+\left\langle\left(\Delta \hat{X}_{\vec{d}^{\prime}}\right)^{2}\right\rangle \geqslant\left|d_{1} d_{2}^{\prime}-d_{2} d_{1}^{\prime}\right|+\left|d_{3} d_{4}^{\prime}-d_{4} d_{3}^{\prime}\right|,
$$

such that for the right combination of $\vec{d}$ and $\vec{d}^{\prime}$ with $\left|d_{i}\right|=$ $\left|d_{i}^{\prime}\right|=1 \forall i$, the first relation is bounded from below by zero, and the second one can be bounded by four.

The uncertainty on the left-hand side can be expressed as

$$
\begin{aligned}
\left\langle\left(\Delta \hat{X}_{\vec{d}}\right)^{2}\right\rangle= & \left|\lambda_{1}\right|^{2}\left(2\left\langle\hat{a}^{\dagger} \hat{a}\right\rangle+1\right)+\left|\lambda_{2}\right|^{2}\left(2\left\langle\hat{b}^{\dagger} \hat{b}\right\rangle+1\right) \\
& +4 \operatorname{Re}\left(\lambda_{1} \lambda_{2}\left\langle\hat{a}^{\dagger} \hat{b}^{\dagger}\right\rangle\right),
\end{aligned}
$$

where $\lambda_{1}=\left(d_{1}+i d_{2}\right) / \sqrt{2}$ and $\lambda_{2}=\left(d_{3}+i d_{4}\right) / \sqrt{2}$, and where the other quadratic terms evaluate to zero for the state given by Eq. (2).

By imposing that $\left|\lambda_{i}\right|=\left|\lambda_{i}^{\prime}\right|=1$ we can find the optimal criterion for separability. Next, without a loss of generality we can set $\operatorname{Arg}\left(\lambda_{1}\right)=0$, impose the saturation of the lowest possible bound of the Heisenberg uncertainty principle by setting $d_{1} d_{2}^{\prime}-d_{2} d_{1}^{\prime}+d_{3} d_{4}^{\prime}-d_{4} d_{3}^{\prime}=0$, and maximize $\left|d_{1} d_{2}^{\prime}-d_{2} d_{1}^{\prime}\right|+\left|d_{3} d_{4}^{\prime}-d_{4} d_{3}^{\prime}\right|=4$, which requires $\operatorname{Arg}\left(\lambda_{1}^{\prime}\right)=$ $\operatorname{Arg}\left(\lambda_{2}\right)-\operatorname{Arg}\left(\lambda_{2}^{\prime}\right)=\frac{\pi}{2}$. This way we turn the separability condition (7) into

$$
4 \leqslant \frac{4\left(1-f_{3}^{2}+|z|^{2}+2|z| \cos \zeta\right)}{\left[1-\left(f_{3}+|z|\right)\right]\left[1-\left(f_{3}-|z|\right)\right]},
$$

where $z=e^{-i \varphi}\left(f_{12}+i f_{5}\right)$ and where we have assumed identical baths $\kappa_{1}=\kappa_{2}$ and $n_{\text {th }, 1}=n_{\text {th, } 2}$ implying $f_{9}=0$ (see discussion in Appendix A2).

This criterion has one left degree of freedom $\zeta=\operatorname{Arg}\left(\lambda_{2}\right)-$ $\operatorname{Arg}(z)$. In these notations, the separability condition simplifies 
to

$$
|z|^{2}+|z| \cos \zeta>f_{3}\left(f_{3}+1\right) .
$$

For $\zeta=0$, we restore the same condition $f_{3}<|z|$ as the one obtained from the condition that the negativity is zero (5). For an arbitrary value of the phase $\zeta$, the separability criterion is generally weaker than the condition that the negativity is zero.

In the next sections, we will use this result in combination with the solutions to the equations of motion to determine the system parameters separability condition.

\section{IMPLICATIONS OF THE SEPARABILITY CONDITIONS}

In order to understand the separability condition in terms of the system parameters, we need to first translate the master equation (1) into a set of equations for functions $f_{i}(t)$ with the initial condition $f_{i}(0)=0 \forall i$. The details of the procedure are outlined in Appendix A4, where we show how an entire set of 15 functions can be obtained. In this work so far we have focused on systems initialized in vacuum state $\rho(0)=|00\rangle\langle 00|$, allowing us to narrow our interest to but four functions, which independent of the initial condition $\rho(0)$, obey the following set of equations:

$$
\begin{aligned}
\dot{f}_{3}= & -\frac{1}{2} c_{21,+} f_{3}-\frac{1}{2} c_{21,-} f_{9}+c_{11,-} f_{3} f_{9}-\frac{1}{2}|\xi| f_{3} f_{5} \\
+ & \frac{1}{2} c_{11,+}\left(f_{3}^{2}+f_{5}^{2}+f_{9}^{2}+f_{12}^{2}\right)+c_{10,+} \\
\dot{f}_{5}= & -\frac{1}{2} c_{21,+} f_{5}+c_{11,+} f_{3} f_{5}+c_{11,-} f_{5} f_{9}+2 \delta f_{12} \\
& +\frac{1}{4}|\xi|\left(-f_{3}^{2}-f_{5}^{2}+f_{9}^{2}+f_{12}^{2}+1\right) \\
\dot{f}_{9}= & -\frac{1}{2} c_{21,-} f_{3}-\frac{1}{2} c_{21,1+} f_{9}+c_{11,+} f_{3} f_{9}-\frac{1}{2}|\xi| f_{5} f_{9} \\
+ & \frac{1}{2} c_{11,-}\left(f_{3}^{2}-f_{5}^{2}+f_{9}^{2}-f_{12}^{2}\right)+\frac{1}{2} c_{10,-}, \\
\dot{f}_{12}= & -\frac{1}{2} c_{21,+} f_{12}+c_{11,+} f_{3} f_{12}+c_{11,-} f_{9} f_{12}-2 \delta f_{5} \\
& -\frac{1}{2}|\xi| f_{5} f_{12}
\end{aligned}
$$

where we have defined

$$
c_{x y, \pm}=\kappa_{1}\left(x n_{\mathrm{th}, 1}+y\right) \pm \kappa_{2}\left(x n_{\mathrm{th}, 2}+y\right) .
$$

It is easy to see that for an identical baths case $\kappa_{1}=\kappa_{2}=\kappa$ and $n_{\mathrm{th}, 1}=n_{\mathrm{th}, 2}$, all $c_{x y,-}=0$, implying that $f_{9}(t)=0$, and completely independently in the resonant regime $\delta=0$ we have $f_{12}(t)=0$. Moreover, in the absence of dissipation, all $c_{x y, \pm}=0$ both $f_{3}(t)$ and $f_{9}(t)$ vanish, and for $\xi=0$ we get $f_{5}(t)=f_{12}(t)=0$. All of these are examples of parameter and Lie algebra reductions leading to significant simplifications in the equations above, to the extent that the nonlinear set of equations above can be solved analytically in a resonant identical baths case, and we were able to analytically determine the steady-state solutions if either the baths are identical, or the system is driven resonantly, or both with $n_{\text {th }, 1}=n_{\text {th }, 2}=0$.

The solutions to the equations above fall into two parameter regimes with a baths population independent boundary

$$
\Xi^{2}=\left[1-\frac{\left(\kappa_{1}-\kappa_{2}\right)^{2}}{\left(\kappa_{1}+\kappa_{2}\right)^{2}}\right]\left[\delta^{2}+\left(\kappa_{1}+\kappa_{2}\right)^{2}\right] .
$$

As a result, we define the underdamped $\left(|\xi|^{2} \geqslant \Xi^{2}\right)$ and overdamped $\left(|\xi|^{2}<\Xi^{2}\right)$ regimes, which we call this way due to either unbounded or bounded expectation values $\left\langle\hat{a}^{\dagger} \hat{a}\right\rangle$ and $\left\langle\hat{b}^{\dagger} \hat{b}\right\rangle$, respectively. Note that the boundary is independent of $n_{\text {th, } 1}$ and $n_{\text {th, } 2}$.

One can verify numerically that both in the overdamped and the underdamped regimes [Eqs. (8)-(11)] possess steadystate solutions. By setting the left-hand sides of these four equations to zero one can obtain the steady-state values of $f_{3}, f_{5}, f_{9}$, and $f_{12}$ algebraically. The process yields a set of solutions larger than those obtained by considering the set of nonlinear ordinary differential equations with initial conditions $f_{i}(0)=0$, therefore, the algebraic solutions found have been verified by solving the differential equations numerically. The steady-state solutions to the equations in the overdamped regime results at zero bath temperature take the compact form

$$
\begin{aligned}
f_{3} & =\frac{|\xi|^{2}\left(\kappa_{1}^{2}+\kappa_{2}^{2}\right)}{8 \kappa_{1} \kappa_{2}\left[\delta^{2}+\left(\kappa_{1}+\kappa_{2}\right)^{2}\right]-2 \kappa_{1} \kappa_{2}|\xi|^{2}}, \\
f_{5} & =\frac{2|\xi|\left(\kappa_{1}+\kappa_{2}\right)}{4\left[\delta^{2}+\left(\kappa_{1}+\kappa_{2}\right)^{2}\right]-|\xi|^{2}}, \\
f_{9} & =\frac{|\xi|^{2}\left(\kappa_{2}^{2}-\kappa_{1}^{2}\right)}{8 \kappa_{1} \kappa_{2}\left[\delta^{2}+\left(\kappa_{1}+\kappa_{2}\right)^{2}\right]-2 \kappa_{1} \kappa_{2}|\xi|^{2}}, \\
f_{12} & =-\frac{2 \delta|\xi|}{4\left[\delta^{2}+\left(\kappa_{1}+\kappa_{2}\right)^{2}\right]-|\xi|^{2}},
\end{aligned}
$$

and the solutions in the $|\xi|^{2}>\Xi^{2}$ regime are too incomprehensible to present here, which is why we will also present the parameter simplified ones. The solutions in the detuned regime with identical nonzero temperature baths read as

$$
\begin{aligned}
f_{3} & =\frac{2 \kappa\left(2 n_{\mathrm{th}}+1\right)}{\sqrt{|\xi|^{2}-\delta^{2}}+4 \kappa\left(n_{\mathrm{th}}+1\right)}, \\
|z| & =\frac{\sqrt{|\xi|^{2}-\delta^{2}}+2 \kappa}{\sqrt{|\xi|^{2}-\delta^{2}}+4 \kappa\left(n_{\mathrm{th}}+1\right)}
\end{aligned}
$$

in the underdamped regime, and in the overdamped regime they become

$$
\begin{aligned}
f_{3} & =\frac{|\xi|^{2}+4 n_{\mathrm{th}}\left(n_{\mathrm{th}}+1\right)\left(\delta^{2}+4 \kappa^{2}\right)}{4\left(n_{\mathrm{th}}+1\right)^{2}\left(\delta^{2}+4 \kappa^{2}\right)-|\xi|^{2}}, \\
|z| & =\frac{2|\xi| \sqrt{\delta^{2}+4 \kappa^{2}}\left(2 n_{\mathrm{th}}+1\right)}{4\left(n_{\mathrm{th}}+1\right)^{2}\left(\delta^{2}+4 \kappa^{2}\right)-|\xi|^{2}} .
\end{aligned}
$$

The two sets of solutions above imply that the separability condition $f_{3}>|z|$ reduces to

$$
\begin{gathered}
4 n_{\mathrm{th}} \kappa>\sqrt{|\xi|^{2}-\delta^{2}} \text { for }|\xi|^{2} \geqslant 4 \kappa^{2}+\delta^{2}, \\
2 n_{\mathrm{th}} \sqrt{4 \kappa^{2}+\delta^{2}}>|\xi| \text { for }|\xi|^{2}<4 \kappa^{2}+\delta^{2},
\end{gathered}
$$

where the parameter regime discontinuity in this result is gone in the absence of detuning, and the same form is obeyed in both the underdamped and the overdamped regimes, where negativity is described by a single function independent of the parameter regime. 
Moreover, in this symmetric resonant regime, where one only needs to consider the solutions to the equations of motion for functions $f_{1-6}$, the other ones returning $f_{7-15}(t)=0$, one can solve the complete set of differential equations analytically also in the transient regime. This has to do with the fact that the master equation is described by a set of operators spanning the so(2,2) Lie algebra, which decomposes into two sets of su(1,1) Lie algebras, with the set of six equations decoupling into two sets of three equations which independently can be solved by the method of quadratures. Identical separability conditions and the same expression for negativity

$$
N=\operatorname{Max}\left[\frac{2\left(1-e^{-t(\kappa+|\xi| / 2)}\right)\left(|\xi|-4 \kappa n_{t h}\right)}{e^{-t(\kappa+|\xi| / 2)}\left(|\xi|-4 \kappa n_{t h}\right)+2 \kappa\left(2 n_{t h}+1\right)}, 0\right]
$$

in either underdamped and overdamped regimes is reflected by the fact that only one of the copies of the $\mathrm{su}(1,1) \mathrm{Lie}$ algebras determines the entanglement. Equation (14) shows that at $t=0$ the state is completely separable, i.e., $N=0$, while in the steady state it is inseparable provided that $|\xi|>$ $4 \kappa n_{t h}$, which is a parameter reduced expression (12) and (13). Moreover, at zero bath temperature, i.e., $n_{\text {th }}=0$ states violate the separability condition in all parameter regimes. Lastly, it is worth observing that in the absence of dissipation $f_{5}=$ $\tanh |\xi| t / 4$ and all other $f_{i}=0$, which not only violates the separability condition for any $t>0$ but also gives rise to a divergent negativity as $t \rightarrow \infty$.

\section{SYSTEM INITIALLY IN A THERMAL STATE}

Since the temperature of the bath plays an important role in the separability condition, it is worth investigating the effect of the initial state's temperature on the steady-state entanglement obtained. In the previous section, we have assumed that the system is initiated in the vacuum state $|00\rangle$, however, in the presence of the environment at a nonzero temperature, this might be difficult to accomplish, and the state prior to two-modesqueeze driving should be initiated in a separable state $\rho(0)=$ $\rho_{\hat{a}, t h} \otimes \rho_{\hat{b}, t h}$, where we denote the thermal-state density operators $\rho_{\hat{c}, t h}=\exp \left[\hbar \omega_{\hat{c}} \hat{c}^{\dagger} \hat{c} / k_{b} T_{\hat{c}}\right] / \operatorname{Tr}\left(\exp \left[\hbar \omega_{\hat{c}} \hat{c}^{\dagger} \hat{c} / k_{b} T_{\hat{c}}\right]\right)$. Here, we will treat the simplest case of the initial condition already in equilibrium with the environment such that $T_{\hat{a}}=T_{\hat{b}}$, $\omega_{\hat{a}}=\omega_{\hat{b}}=\omega$, and hence $n_{t h, 1}=n_{t h, 2}=\tau(1-\tau)^{-1}$, with $\tau=e^{-\beta \hbar \omega}$. The two modes still remain orthogonal, i.e., $\hat{a} \neq \hat{b}$. The initial condition now can be written as $\rho(0)=\rho_{\hat{a}, t h} \otimes$ $\rho_{\hat{b}, t h}=(1-\tau)^{2} e^{\tau H_{3}}|00\rangle\langle 00|$. With the suitably chosen normal ordered solution ansatz

$$
\begin{aligned}
\rho(t)= & e^{f_{0}(t)} e^{f_{3}(t) H_{3}} e^{f_{5}(t) H_{5}} e^{f_{1}(t) H_{1}} e^{f_{6}(t) H_{6}} \\
& \times e^{f_{2}(t) H_{2}} e^{f_{4}(t) H_{4}} \rho(0),
\end{aligned}
$$

where operators $H_{1,2,4,6}$ contain normal ordered annihilation operators. We see that since the system is no longer initiated in the vacuum, we cannot disregard a given set of exponents of operators. Using the form of the initial condition

$$
\begin{aligned}
\rho(t)= & (1-\tau)^{2} e^{f_{0}(t)} e^{f_{3}(t) H_{3}} e^{f_{5}(t) H_{5}} e^{f_{1}(t) H_{1}} e^{f_{6}(t) H_{6}} \\
& \times e^{f_{2}(t) H_{2}} e^{f_{4}(t) H_{4}} e^{\tau H_{3}}|00\rangle\langle 00|
\end{aligned}
$$

we can now commute $\exp \left[\tau \mathrm{H}_{3}\right]$ through exponents of operators $H_{1,2,4,6}$, and redecompose using the Wei-Norman scheme as presented in the Appendix. As a result, we obtain

$$
\rho(t)=\left[\left(1-g_{3}\right)^{2}-g_{5}^{2}\right] e^{g_{3}(t) H_{3}} e^{g_{5} H_{5}}|00\rangle\langle 00|,
$$

where $g_{i}(t)=f_{i}(t)+\mathcal{F}_{i}(t)$, and where the only two relevant $\mathcal{F}_{i}$ as functions of $f_{i}$ read as

$$
\begin{aligned}
& \mathcal{F}_{3}(t)=\frac{1}{2}\left(-\frac{e^{2 f_{1}+f_{6}} \tau}{f_{2} \tau-f_{4} \tau-1}-\frac{e^{2 f_{1}-f_{6}} \tau}{f_{2} \tau+f_{4} \tau-1}\right), \\
& \mathcal{F}_{5}(t)=\frac{1}{2}\left(\frac{e^{2 f_{1}-f_{6}} \tau}{f_{2} \tau+f_{4} \tau-1}-\frac{e^{2 f_{1}+f_{6} \tau}}{f_{2} \tau-f_{4} \tau-1}\right) .
\end{aligned}
$$

Note that unlike before, here the knowledge of functions $f_{1}, f_{2}, f_{4}$, and $f_{6}$ is needed. By virtue of the form of Eq. (15), we can reuse our previous result and replace $f_{3,5}$ with $g_{3,5}$, and we can immediately state that negativity will take the form

$$
N=\operatorname{Max}\left[\frac{\left(|\xi|-4 \kappa n_{t h}\right)-e^{-t(\kappa+|\xi| / 2)}|\xi|\left(2 n_{t h}+1\right)}{2\left(2 n_{t h}+1\right)\left(|\xi| e^{-t(\kappa+|\xi|)}+2 \kappa\right)}, 0\right]
$$

Moreover, this result in comparison to Eq. (14) has the same steady-state amount of entanglement $\frac{|\xi|-4 \kappa n_{t h}}{4 \kappa\left(2 n_{t h}+1\right)}$, however, the key difference is that when the state starts in a thermal equilibrium with the environment its negativity remains zero for a finite amount of time $t=2(2 \kappa+|\xi|)^{-1} \ln \left(\frac{|\xi|\left(2 n_{t h}+1\right) / 2}{|\xi|-4 \kappa n_{t h}}\right)$, which only makes sense for the case of any entanglement formed, i.e., $|\xi|>4 \kappa n_{\mathrm{th}}$.

\section{CONCLUSIONS}

In this work, we have shown that one can use the WeiNorman method to study analytically a bosonic entanglement process subject to dissipation. The Lie algebra valued description based solution ansatz allows one to calculate the exact expression of entanglement evolution or its steady-state form as measured by negativity. Additionally, we have shown that the negativity calculated from the solution is completely compatible with the continuous variable separability condition. Moreover, we have shown that for time-independent system parameters, one can determine analytically the solutions to the equations of motion in the Wei-Norman setting in the transient and the steady state. Finally, should the bipartite state be initially in thermal equilibrium with the environment, then the steady-state entanglement does not change, however, there is a finite amount of time in the transient regime where the degree of entanglement is lower compared to that when the state is initialized in vacuum.

The results formulated in this paper in terms of general functions $f_{i}$ remain applicable for (effective) two-mode driven systems with time-dependent parameters (driving strength $\xi$, dissipation rates $\kappa_{i}$, or detuning $\delta$ ), which then require using the same equations with time-dependent coefficients. Moreover, this method is very well suited for investigating similar problems of more than two modes with pairwisesqueezing interaction terms. As a result, such extensions can be very important in experiments investigating entanglement in continuous variable systems.

A standard system where our theory can be applied is a parametric amplifier, in which two quantum modes are 
classically pumped by a third mode [24]. Recently, we also used the two-mode-squeezing technique developed above for a parametrically driven mechanical resonator with two nonlinearly coupled modes [4]. When they are in the quantum regime, the relevant interaction terms look indeed like twomode-squeezing terms. However, the quantum regime for the two interacting mechanical modes has not been yet achieved experimentally.

Another system suitable for application of our theory may be cavity and circuit optomechanics. In optomechanical schemes, electromagnetic radiation is coupled to a mechanical resonator (see Ref. [3] for review). For strong cavity driving (known as multiphoton regime), the interaction between photons in the cavity $(\hat{a})$ and phonons in the mechanical resonator $(\hat{b})$ can be linearized and contains all four linear combinations $\left(\hat{a}^{\dagger} \hat{b}, \hat{a} \hat{b}^{\dagger}, \hat{a} \hat{b}\right.$, and $\left.\hat{a}^{\dagger} \hat{b}^{\dagger}\right)$. The last two terms which give squeezing are counter-rotating and are typically disregarded. However, if the coupling between phonons and photons is not negligible in comparison with the mechanical frequency (which is typically much lower than the frequency of the cavity), these terms need to be taken into account. Our paper suggests a possible way of dealing with these terms and investigating the entanglement to all orders between the cavity and the resonator, an important feature characterizing quantum properties of phonons.

\section{ACKNOWLEDGMENTS}

The authors wish to thank G. Labadze and A. Borrás for useful discussions. This work was supported by the Foundation for Fundamental Research on Matter (FOM).

\section{APPENDIX}

\section{Lindblad equation of motion and the underlying Lie algebra structure}

The Lindblad-type master equation (1) can be rewritten in the form

$$
\dot{\rho}=\sum_{i=0}^{15} \alpha_{i} H_{i} \rho,
$$

where

$$
\begin{aligned}
\alpha_{1} & =-\frac{1}{4}\left[\kappa_{1}\left(2 n_{1, t h}+1\right)+\kappa_{2}\left(2 n_{2, t h}+1\right)\right], \\
\alpha_{2,8} & =\frac{1}{2}\left[\kappa_{1}\left(n_{1, t h}+1\right) \pm \kappa_{2}\left(n_{2, t h}+1\right)\right], \\
\alpha_{7} & =-\frac{1}{2}\left[\kappa_{1}\left(2 n_{1, t h}+1\right)-\kappa_{2}\left(2 n_{2, t h}+1\right)\right], \\
\alpha_{3,9} & =\frac{1}{2}\left(\kappa_{1} n_{1, t h} \pm \kappa_{2} n_{2, t h}\right), \quad \alpha_{0}=\frac{1}{2}\left(\kappa_{1}+\kappa_{2}\right), \\
\alpha_{4} & =\alpha_{5}=\frac{1}{4}|\xi|, \quad \alpha_{15}=2 \delta,
\end{aligned}
$$

with all other $\alpha_{i}=0$, and where

$$
\begin{aligned}
H_{1,7} \rho= & \frac{1}{2}\left(\hat{a} \hat{a}^{\dagger} \rho+\hat{a}^{\dagger} \hat{a} \rho+\rho \hat{a} \hat{a}^{\dagger}+\rho \hat{a}^{\dagger} \hat{a}\right) \\
& \pm \frac{1}{2}\left(\hat{b} \hat{b}^{\dagger} \rho+\hat{b}^{\dagger} \hat{b} \rho+\rho \hat{b} \hat{b}^{\dagger}+\rho \hat{b}^{\dagger} \hat{b}\right), \\
H_{2,8} \rho= & \hat{a} \rho \hat{a}^{\dagger} \pm \hat{b} \rho \hat{b}^{\dagger}, \\
H_{3,9} \rho= & \hat{a}^{\dagger} \rho \hat{a} \pm \hat{b}^{\dagger} \rho \hat{b}, \\
H_{4,11} \rho= & e^{i[\varphi+(1 \pm 1) \pi / 4]} \hat{a} \hat{b} \rho-e^{-i[\varphi+(1 \pm 1) \pi / 4]} \rho \hat{a}^{\dagger} \hat{b}^{\dagger},
\end{aligned}
$$

$$
\begin{aligned}
H_{5,12} \rho & =e^{-i[\varphi-(1 \pm 1) \pi / 4]} \hat{a}^{\dagger} \hat{b}^{\dagger} \rho-e^{i[\varphi-(1 \pm 1) \pi / 4]} \rho \hat{a} \hat{b}, \\
H_{6,10} \rho & =\frac{1}{2} i\left(e^{-i \varphi} \hat{b}^{\dagger} \rho \hat{a}^{\dagger}-e^{i \varphi} \hat{a} \rho \hat{b} \mp e^{i \varphi} \hat{b} \rho \hat{a} \pm e^{-i \varphi} \hat{a}^{\dagger} \rho \hat{b}^{\dagger}\right), \\
H_{13,14} \rho & =\frac{1}{2}\left(e^{i \varphi} \hat{a} \rho \hat{b} \pm e^{i \varphi} \hat{b} \rho \hat{a} \pm e^{-i \varphi} \hat{a}^{\dagger} \rho \hat{b}^{\dagger}+e^{-i \varphi} \hat{b}^{\dagger} \rho \hat{a}^{\dagger}\right), \\
H_{15} \rho & =\frac{1}{2} i\left(\hat{a}^{\dagger} \hat{a} \rho-\rho \hat{a}^{\dagger} \hat{a}+\hat{b}^{\dagger} \hat{b} \rho-\rho \hat{b}^{\dagger} \hat{b}\right),
\end{aligned}
$$

with the first (second) index corresponding to the upper (lower) signs and with $H_{0}$ being the identity superoperator, i.e., $H_{0} \rho=\rho$.

Thanks to the elementary commutation relation

$$
\left[\Theta_{i}, \Theta_{j}^{\dagger}\right]=\delta_{i, j} \quad \text { and } \quad\left[\Theta_{i}, \Theta_{j}\right]=0 \text {, }
$$

where $\Theta_{1}=\hat{a}$ and $\Theta_{2}=\hat{b}$, the set of 15 superoperators closes under commutation (see Table I), thus forming a Lie algebra. In what follows, we define skew (anti)symmetric matrices

$$
L_{i, j}=E_{i, j}+E_{j, i}, \quad K_{i, j}=E_{i, j}-E_{j, i},
$$

where $E_{i, j}$ is a matrix with 1 in the $i$ th row and $j$ th column and zero elsewhere. It is easy to verify that the linear combinations of the above

$$
\begin{aligned}
\mathcal{H}_{1} & =-2 L_{2,3}, \quad \mathcal{H}_{6}=L_{1,5}, \quad \mathcal{H}_{7}=-L_{1,4}, \\
\mathcal{H}_{10} & =K_{4,5}, \quad \mathcal{H}_{13}=L_{1,6}, \quad \mathcal{H}_{14}=K_{5,6}, \\
\mathcal{H}_{15} & =K_{4,6}
\end{aligned}
$$

and

$$
\begin{array}{ll}
\mathcal{H}_{2,3}=K_{1,2} \mp L_{1,3}, & \mathcal{H}_{4,5}=L_{2,5} \pm K_{3,5}, \\
\mathcal{H}_{8,9}=K_{3,4} \pm L_{2,4}, & \mathcal{H}_{11,12}=-K_{3,6} \mp L_{2,6}
\end{array}
$$

obey the same commutation relations, and that the $L_{i, j}$ and $K_{i, j}$ above are elements of the so(4,2) Lie algebra. Upon a homomorphism $H_{i} \rightarrow \mathcal{H}_{i}$ we can show that the superoperators from the master equation (1) are just a different incarnation of the so(4,2) Lie algebra.

Realizing that, for time independent $\kappa_{i}, n_{t h, i}, \delta$, and $\xi$, the solution to the master equation (1) in the form of (A1) is simply

$$
\rho(t)=\exp \left[t \sum_{i=0}^{15} \alpha_{i} H_{i}\right] \rho(0),
$$

which is simply given by a Lie group element acting on the initial state. This can be thought of as a rotation, or a movement on the surface embedded in six dimensions satisfying the equation

$$
1=-x_{1}^{2}-x_{2}^{2}+x_{3}^{2}+x_{4}^{2}+x_{5}^{2}+x_{6}^{2},
$$

which can be understood as the trace-preservation condition of the density operator $\rho$ [25]. The form of Eq. (A3), however, is not very useful for any purposes, and we will proceed with the so called Wei-Norman method [7,8], to decompose the right-hand side of Eq. (A3), however, this method is much more powerful and allows one to solve Eq. (1) for time dependent 
TABLE I. Commutation relation table. The smallest (middle) $6 \times 6(10 \times 10)$ box encloses the so(2,2) [so(3,2)] Lie algebra.

\begin{tabular}{|c|c|c|c|c|c|c|c|c|c|c|c|c|c|c|c|}
\hline$[\ldots, \ldots]$ & $H_{1}$ & $\mathrm{H}_{2}$ & $\mathrm{H}_{3}$ & $\mathrm{H}_{4}$ & $H_{5}$ & $H_{6}$ & $H_{7}$ & $H_{8}$ & $H_{9}$ & $H_{10}$ & $H_{11}$ & $H_{12}$ & $H_{13}$ & $H_{14}$ & $H_{15}$ \\
\hline$H_{1}$ & 0 & $-2 \mathrm{H}_{2}$ & $2 \mathrm{H}_{3}$ & $-2 \mathrm{H}_{4}$ & $2 \mathrm{H}_{5}$ & 0 & 0 & $-2 H_{8}$ & $2 \mathrm{H}_{9}$ & 0 & $-2 H_{11}$ & $2 H_{12}$ & 0 & 0 & 0 \\
\hline $\mathrm{H}_{2}$ & $2 \mathrm{H}_{2}$ & 0 & $H_{1}$ & 0 & $2 \mathrm{H}_{6}$ & $-H_{4}$ & $H_{8}$ & 0 & $2 \mathrm{H}_{7}$ & 0 & 0 & $2 H_{13}$ & $H_{11}$ & 0 & 0 \\
\hline $\mathrm{H}_{3}$ & $-2 \mathrm{H}_{3}$ & $-H_{1}$ & 0 & $2 \mathrm{H}_{6}$ & 0 & $-H_{5}$ & $-H_{9}$ & $-2 H_{7}$ & 0 & 0 & $-2 H_{13}$ & 0 & $-H_{12}$ & 0 & 0 \\
\hline$H_{4}$ & $2 \mathrm{H}_{4}$ & 0 & $-2 H_{6}$ & 0 & $-H_{1}$ & $-H_{2}$ & 0 & 0 & $2 H_{10}$ & $-H_{8}$ & 0 & $2 H_{15}$ & 0 & 0 & $-H_{11}$ \\
\hline$H_{5}$ & $-2 \mathrm{H}_{5}$ & $-2 H_{6}$ & 0 & $H_{1}$ & 0 & $-H_{3}$ & 0 & $-2 H_{10}$ & 0 & $H_{9}$ & $-2 H_{15}$ & 0 & 0 & 0 & $H_{12}$ \\
\hline$H_{6}$ & 0 & $H_{4}$ & $H_{5}$ & $\mathrm{H}_{2}$ & $\mathrm{H}_{3}$ & 0 & $H_{10}$ & 0 & 0 & $H_{7}$ & 0 & 0 & $H_{15}$ & 0 & $H_{13}$ \\
\hline$H_{7}$ & 0 & $-H_{8}$ & $H_{9}$ & 0 & 0 & $-H_{10}$ & 0 & $-H_{2}$ & $H_{3}$ & $-H_{6}$ & 0 & 0 & $-H_{14}$ & $-H_{13}$ & 0 \\
\hline$H_{8}$ & $2 H_{8}$ & 0 & $2 \mathrm{H}_{7}$ & 0 & $2 H_{10}$ & 0 & $H_{2}$ & 0 & $H_{1}$ & $H_{4}$ & 0 & $2 H_{14}$ & 0 & $-H_{11}$ & 0 \\
\hline$H_{9}$ & $-2 H_{9}$ & $-2 \mathrm{H}_{7}$ & 0 & $-2 H_{10}$ & 0 & 0 & $-H_{3}$ & $-H_{1}$ & 0 & $-H_{5}$ & $2 H_{14}$ & 0 & 0 & $-H_{12}$ & 0 \\
\hline$H_{10}$ & 0 & 0 & 0 & $H_{8}$ & $-H_{9}$ & $-H_{7}$ & $H_{6}$ & $-H_{4}$ & $H_{5}$ & 0 & 0 & 0 & 0 & $-H_{15}$ & $H_{14}$ \\
\hline$H_{11}$ & $2 H_{11}$ & 0 & $2 H_{13}$ & 0 & $2 H_{15}$ & 0 & 0 & 0 & $-2 H_{14}$ & 0 & 0 & $H_{1}$ & $\mathrm{H}_{2}$ & $H_{8}$ & $\mathrm{H}_{4}$ \\
\hline$H_{12}$ & $-2 H_{12}$ & $-2 H_{13}$ & 0 & $-2 H_{15}$ & 0 & 0 & 0 & $-2 H_{14}$ & 0 & 0 & $-H_{1}$ & 0 & $-H_{3}$ & $H_{9}$ & $-H_{5}$ \\
\hline$H_{13}$ & 0 & $-H_{11}$ & $H_{12}$ & 0 & 0 & $-H_{15}$ & $H_{14}$ & 0 & 0 & 0 & $-\mathrm{H}_{2}$ & $\mathrm{H}_{3}$ & 0 & $H_{7}$ & $-H_{6}$ \\
\hline$H_{14}$ & 0 & 0 & 0 & 0 & 0 & 0 & $H_{13}$ & $H_{11}$ & $H_{12}$ & $H_{15}$ & $-H_{8}$ & $-H_{9}$ & $-H_{7}$ & 0 & $-H_{10}$ \\
\hline$H_{15}$ & 0 & 0 & 0 & $H_{11}$ & $-H_{12}$ & $-H_{13}$ & 0 & 0 & 0 & $-H_{14}$ & $-H_{4}$ & $H_{5}$ & $H_{6}$ & $H_{10}$ & 0 \\
\hline
\end{tabular}

$\kappa_{i}, n_{t h, i}, \delta$, and $\xi$, allowing for studying modulated squeezedriving and non-Markovian baths.

\section{Wei-Norman method treatment and the resultant equations of motion}

We take the ansatz

$$
\rho(t)=e^{f_{0}(t)} \prod_{i} e^{f_{i}(t) H_{i}} \rho(0)
$$

with the ordering $i=3,5,9,12,1,7,15,6,14,10,13,2,8,4,11$, with the exponent of $H_{3}$ acting last and the exponent of $H_{11}$ acting first on the initial condition $\rho(0)$. From the definition of the superoperators $H_{i}$, one can see that the ordering chosen above is normal, i.e., annihilation (creation) superoperators acting first (last), and in the middle superoperators which are composed of creation and annihilation operators. ${ }^{3}$ Lastly, it is important to note that a different ordering ansatz will result in a different set of equations for functions $f_{i}(t)$.

The set of resultant differential equations is quite complicated and nontransparent, thus outside the scope of this article. In a special, very convenient, case when $\rho(0)=|00\rangle\langle 00|$, we see that the set of 11 rightmost operators acting on the initial condition leaves it unchanged, with the exception of $e^{f_{1} H_{1}}$ which contributes an overall scaling factor, thus in combination with the only decoupled equation of motion $\dot{f}_{0}=\frac{1}{2}\left(\kappa_{1}+\kappa_{2}\right)$, gives

$$
\begin{aligned}
\rho(t) & =e^{\frac{1}{2}\left(\kappa_{1}+\kappa_{2}\right) t+2 f_{1}} e^{f_{3}(t) H_{3}} e^{f_{5}(t) H_{5}} e^{f_{9}(t) H_{9}} e^{f_{12}(t) H_{12}}|00\rangle\langle 00 \\
& \equiv e^{\frac{1}{2}\left(\kappa_{1}+\kappa_{2}\right) t+2 f_{1}} \tilde{\rho},
\end{aligned}
$$

\footnotetext{
${ }^{3}$ The ordering of the indices might not seem very natural, however, here we had to make a choice between transparent Lie subalgebra division (discussed later) and renaming of the superoperators such that the ordering is $i=1,2, \ldots, 15$; in this work we chose the former.
}

at which point the order does not matter due to the mutual commutativity of the remaining operators, and the scalar prefactor plays the role of a normalization condition. Regardless of the initial condition, the kinetic equations for functions $f_{3}, f_{5}, f_{9}$, and $f_{12}$ were given in the main body of the text and the necessary equation for $f_{1}$ takes the form

$$
\dot{f}_{1}=\frac{1}{2} c_{11,+} f_{3}+\frac{1}{2} c_{11,-} f_{9}-\frac{1}{2}|\xi| f_{5}-\frac{1}{4} c_{21,+} .
$$

Using the set of equations (8)-(11) and the equation above, one can verify by differentiating both sides and remembering the initial condition $f_{i}(t=0)=0 \forall i$, that

$$
\frac{e^{2 f_{1}+\frac{1}{2} t\left(\kappa_{1}+\kappa_{2}\right)}}{\left(1-f_{3}\right)^{2}-f_{5}^{2}-f_{9}^{2}-f_{12}^{2}}=1,
$$

which allows us to eliminate the scale factor in Eq. (A5) in favor of functional dependence on functions $f_{3}, f_{5}, f_{9}$, and $f_{12}$. One can verify the trace-preserving nature of the evolution by taking the trace of the equation and arriving at $\partial_{t} \operatorname{Tr}(\rho)=0$.

Additionally, the tabular display of the operators into subgroups marks the use of smaller Lie algebras equation decompositions, and surface-dimensional reduction, such that we have the following:

1. $\delta=0$ decouples operators $H_{11-15}$ from the algebra $\left[H_{15}\right.$ vanishes directly from the equation of motion, and $H_{11-14}$ do not enter the dynamics due to their commutation relation properties (see Table I)].

2. $\kappa_{1}=\kappa_{2}=\kappa \neq 0$ and $n_{t h, 1}=n_{t h, 2}$, decouples operators $H_{7-9}$, and effectively the operators $H_{10}$ and $H_{14}$, and the system reduces again to $\operatorname{so}(3,2) \subset \operatorname{so}(4,2)$.

3. $\xi=0$, decouples operators $H_{4,5}$, and effectively $H_{6,10-15}$. The relevant operators form an $\operatorname{so}(2,2) \subset \operatorname{so}(4,2)$ Lie algebra which decomposes into two copies of $\operatorname{su}(1,1)$ Lie algebras acting in separate subspaces of $\hat{a}$ and $\hat{b}$ bosons.

4. $\kappa_{1}=\kappa_{2}=0$, decouples operators $H_{2,3,6}, H_{7-10}$, and $H_{13,14}$. The remaining operators form again an $\operatorname{so}(2,2) \subset$ so(4,2) Lie algebra which decomposes into two sets of operators acting separately from the right or from the left of the 
density operator. This has to do with the fact that said evolution no longer needs to be described using a von Neumann equation in superoperators, but rather a Schrödinger equation described only by right or left acting operators separately.

5. Moreover, conditions 1 and 2 combined also lead to an so $(2,2)$ reduction, where the decomposition into two copies of $\mathrm{su}(1,1)$ Lie algebras is different.

The Lie-algebraic reduction described above has to do with the reduced dimensionality of the space embedding the surface, to which one can deem the evolution to be confined, such that for the so $(3,2)$ Lie algebra case we are dealing with a five-dimensional space with a surface given by the equation $1=-x_{1}^{2}-x_{2}^{2}+x_{3}^{2}+x_{4}^{2}+x_{5}^{2}$ and in the $\operatorname{so}(2,2) \sim$ $\operatorname{su}(1,1) \otimes \operatorname{su}(1,1) \sim \operatorname{so}(2,1) \otimes \operatorname{so}(2,1)$ the evolution is confined to a product space of two hyperboloids embedded in three dimensions.

\section{Computing the negativity}

The solutions to the equations of motion written using the Wei-Norman method conditioned on both modes initially in the vacuum state are given in Eq. (2). For the purposes of this proof, we will rewrite this result using the property of mutual commutation of the above operators

$$
\begin{aligned}
\rho(t)= & \mathcal{N} e^{\left(f_{3}+f_{9}\right) \hat{a}^{\dagger} \cdot \hat{a}} e^{\left(f_{3}-f_{9}\right) \hat{b}^{\dagger} \cdot \hat{b}} \\
& \times e^{\left(f_{12}+i f_{5}\right) e^{-i \varphi} \hat{a}^{\dagger} \hat{b}^{\dagger}} \cdot e^{\left(f_{12}-i f_{5}\right) e^{i \varphi} \cdot \hat{a} \hat{b}}|00\rangle\langle 00|
\end{aligned}
$$

and we will rewrite the matrix in terms of a quadruple infinite sum with redefinitions $g_{ \pm}=f_{3} \pm f_{9}$ and $z=\left(f_{12}+i f_{5}\right) e^{-i \varphi}$ :

$$
\begin{aligned}
\rho= & \mathcal{N} \sum_{i j k l=0}^{\infty} \frac{g_{+}^{i} g_{-}^{j} z^{k} \bar{z}^{l}}{i ! j ! k ! l !}\left(\hat{a}^{\dagger}\right)^{i+k}\left(\hat{b}^{\dagger}\right)^{j+k}|00\rangle\langle 00| \hat{a}^{i+l} \hat{b}^{j+l} \\
= & \mathcal{N} \sum_{i j k l=0}^{\infty} \frac{g_{+}^{i} g_{-}^{j} z^{k} \bar{z}^{l}}{i ! j ! k ! l !} \sqrt{(i+k) !(j+k) !(i+l) !(j+l) !} \\
& \times|i+k, j+k\rangle\langle i+l, j+l| .
\end{aligned}
$$

In order to calculate the negativity, we need to partial transpose the matrix above, which can be done very easily:

$$
\begin{aligned}
\rho^{p T}= & \mathcal{N} \sum_{i j k l=0}^{\infty} \frac{g_{+}^{i} g_{-}^{j} z^{k} \bar{z}^{l}}{i ! j ! k ! l !} \sqrt{(i+k) !(j+k) !(i+l) !(j+l) !} \\
& \times|i+k, j+l\rangle\langle i+l, j+k| \\
= & \mathcal{N} \sum_{i j k l=0}^{\infty} \frac{g_{+}^{i} g_{-}^{j} z^{k} \bar{z}^{l}}{i ! j ! k ! l !}\left(\hat{a}^{\dagger}\right)^{i+k}\left(\hat{b}^{\dagger}\right)^{j+l}|00\rangle\langle 00| \hat{a}^{i+l} \hat{b}^{j+k} .
\end{aligned}
$$

Next, we use the relationship and define a shorthand $\rho^{p T} \equiv \mathcal{X}$ and the relationships

$$
\operatorname{det}[\exp (A)]=\exp [\operatorname{Tr}(A)] \Rightarrow \operatorname{det}[X]=\exp [\operatorname{Tr}(\ln X)]
$$

to derive the characteristic equation and determine the eigenvalues we use

$$
\begin{aligned}
\operatorname{det}[\mathcal{X}-I \lambda] & =\operatorname{det}[-\lambda I] \operatorname{det}\left[I-\frac{\mathcal{X}}{\lambda}\right] \\
& =\operatorname{det}[-\lambda I] \exp \left\{\operatorname{Tr}\left[\ln \left(I-\frac{\mathcal{X}}{\lambda}\right)\right]\right\} \\
& =\operatorname{det}[-\lambda I] \exp \left[\operatorname{Tr}\left(\sum_{j=1}^{\infty}-\frac{\mathcal{X}}{j \lambda^{j}}\right)\right] \\
& =\operatorname{det}[-\lambda I] \exp \left[\sum_{j=1}^{\infty}-\frac{\operatorname{Tr}\left(\mathcal{X}^{j}\right)}{j \lambda^{j}}\right],
\end{aligned}
$$

where the $\ln$ Taylor expansion holds if the eigenvalues of $X$ observe the condition $\left|\lambda_{i}\right| \leqslant 1$, which is the case for the eigenvalues of any (partial transposed) density operator. If we find a general form of $\operatorname{Tr}\left(X^{j}\right)$, then we can hope to find the form of this infinitely long polynomial. Already when calculating a square of $\mathcal{X}$ we can see a pattern

$$
\mathcal{X}^{2}=\mathcal{N}^{2} \sum_{i j k l=0}^{\infty} \sum_{p q r s=0}^{\infty} \frac{g_{+}^{i} g_{-}^{j} z^{k} \bar{z}^{l}}{i ! j ! k ! l !}\left(\hat{a}^{\dagger}\right)^{i+k}\left(\hat{b}^{\dagger}\right)^{j+l}|00\rangle\left\langle 00\left|\hat{a}^{i+l} \hat{b}^{j+k}\left(\hat{a}^{\dagger}\right)^{p+s}\left(\hat{b}^{\dagger}\right)^{q+r}\right| 00\right\rangle\langle 00| \hat{a}^{p+r} \hat{b}^{q+s} \frac{g_{+}^{p} g_{-}^{q} z^{s} \bar{z}^{r}}{p ! q ! r ! s !} .
$$

Then, focusing on the ket-operator sandwich in the middle we see that

$$
\left\langle 00\left|\hat{a}^{w} \hat{b}^{x}\left(\hat{a}^{\dagger}\right)^{y}\left(\hat{b}^{\dagger}\right)^{z}\right| 00\right\rangle=\delta_{w, y} \delta_{x, z} w ! x !,
$$

in our case implying $p=i+l-s$ and $q=j+k-r$, hence $i+l \geqslant s$ and $j+k \geqslant r$ :

$$
\begin{aligned}
\mathcal{X}^{2} & =\mathcal{N}^{2} \sum_{i j k l=0}^{\infty} \sum_{r=0}^{j+k} \sum_{s=0}^{i+l} \frac{g_{+}^{i} g_{-}^{j} z^{k} \bar{z}^{l}}{i ! j ! k ! l !}\left(\hat{a}^{\dagger}\right)^{i+k}\left(\hat{b}^{\dagger}\right)^{j+l}|00\rangle\langle 00| g_{+}^{i+l-s} g_{-}^{j+k-r} z^{s} \bar{z}^{r} \frac{(i+l) !(j+k) !}{(i+l-s) !(j+k-r) ! r ! s !} \hat{a}^{i+l-s+r} \hat{b}^{j+k-r+s} \\
& =\mathcal{N}^{2} \sum_{i j k l=0}^{\infty} \frac{g_{+}^{i} g_{-}^{j} z^{k} \bar{z}^{l}}{i ! j ! k ! l !}\left(\hat{a}^{\dagger}\right)^{i+k}\left(\hat{b}^{\dagger}\right)^{j+l}|00\rangle\langle 00| \sum_{r=0}^{j+k}\left(\begin{array}{c}
j+k \\
r
\end{array}\right) g_{-}^{j+k-r} \bar{z}^{r} \hat{b}^{j+k-r} \hat{a}^{r} \sum_{s=0}^{i+l}\left(\begin{array}{c}
i+l \\
s
\end{array}\right) g_{+}^{i+l-s} z^{s} \hat{a}^{i+l-s} \hat{b}^{s} \\
& =\mathcal{N}^{2} \sum_{i j k l=0}^{\infty} \frac{g_{+}^{i} g_{-}^{j} z^{k} \bar{z}^{l}}{i ! j ! k ! l !}\left(\hat{a}^{\dagger}\right)^{i+k}\left(\hat{b}^{\dagger}\right)^{j+l}|00\rangle\langle 00|\left(g_{-} \hat{b}+\bar{z} \hat{a}\right)^{j+k}\left(g_{+} \hat{a}+z \hat{b}\right)^{i+l} \\
& =\mathcal{N}^{2} \sum_{i=0}^{\infty} \frac{g_{+}^{i}}{i !}\left[\hat{a}^{\dagger} \cdot\left(g_{+} \hat{a}+z \hat{b}\right)\right]^{i} \sum_{j=0}^{\infty} \frac{g_{-}^{j}}{j !}\left[\hat{b}^{\dagger} \cdot\left(\bar{z} \hat{a}+g_{-} \hat{b}\right)\right]^{j} \sum_{k=0}^{\infty} \frac{z^{k}}{k !}\left[\hat{a}^{\dagger} \cdot\left(\bar{z} \hat{a}+g_{-} \hat{b}\right)\right]^{k} \sum_{l=0}^{\infty} \frac{\bar{z}^{l}}{l !}\left[\hat{b}^{\dagger} \cdot\left(g_{+} \hat{a}+z \hat{b}\right)\right]^{l}|00\rangle\langle 00|
\end{aligned}
$$




$$
\begin{aligned}
& =\mathcal{N}^{2} \exp \left[g_{+} \hat{a}^{\dagger} \cdot\left(g_{+} \hat{a}+z \hat{b}\right)+g_{-} \hat{b}^{\dagger} \cdot\left(\bar{z} \hat{a}+g_{-} \hat{b}\right)+z \hat{a}^{\dagger} \cdot\left(\bar{z} \hat{a}+g_{-} \hat{b}\right)+\bar{z} \hat{b}^{\dagger} \cdot\left(g_{+} \hat{a}+z \hat{b}\right)\right] \\
& =\mathcal{N}^{2} \exp \left\{\left(f_{3}^{2}+f_{5}^{2}+f_{9}^{2}+f_{12}^{2}\right) H_{3}+2 f_{3} f_{9} H_{9}+2 f_{3} \sqrt{f_{5}^{2}+f_{12}^{2}}\left[\sin (\theta+\varphi) H_{5}^{p T}+\cos (\theta+\varphi) H_{12}^{p T}\right]\right\}|00\rangle\langle 00| .
\end{aligned}
$$

Since the trace is unaffected by (partial) transposition, and the trace of $\mathcal{X}^{2} / \mathcal{N}^{2}$ is the same as the trace of $\rho / \mathcal{N}$ with the replacement of $f_{3} \rightarrow f_{3}^{2}+f_{5}^{2}+f_{9}^{2}+f_{12}^{2}, f_{9} \rightarrow 2 f_{3} f_{9}$, and $f_{5}^{2}+f_{12}^{2} \rightarrow 4 f_{3}^{2}\left(f_{5}^{2}+f_{12}^{2}\right)$, giving after simplification

$$
\operatorname{Tr}\left(\mathcal{X}^{2}\right)=\frac{\left(1-x_{+}^{2}\right)\left(1-x_{-}\right)^{2}}{\left(1-x_{+}^{2}\right)\left(1-x_{-}^{2}\right)} .
$$

Following the argument above, it is easy to prove in general (after some algebra) that every additional power of $\mathcal{X}$ gives rise to the transformation $(\ldots) \hat{a} \rightarrow g_{+}(\ldots) \hat{a}+z(\ldots) \hat{b}$ and $\hat{b} \rightarrow \bar{z}(\ldots) \hat{a}+g_{-}(\ldots) \hat{b}$. Using a proof by induction, one can prove that upon tracing $\mathcal{X}^{j}$ we get

$$
\operatorname{Tr}\left(\mathcal{X}^{j}\right)=\frac{\left(1-x_{+}\right)^{j}\left(1-x_{-}\right)^{j}}{\left(1-x_{+}^{j}\right)\left(1-x_{-}^{j}\right)} .
$$

Alternatively, one can see that $\mathcal{X}^{j}$ can always be written in the form

$$
\mathcal{X}^{j}=\mathcal{N}^{j} \exp \left[F_{3}^{(j)} H_{3}+F_{9}^{(j)} H_{9}+F_{5}^{(j)} H_{5}^{p T}+F_{12}^{(j)} H_{12}^{p T}\right]
$$

with $\operatorname{Tr}\left(\mathcal{X}^{j}\right)$ in the form

$$
\operatorname{Tr}\left(\mathcal{X}^{j}\right)=\frac{\mathcal{N}^{j}}{\left(1-X_{+}^{(j)}\right)\left(1-X_{-}^{(j)}\right)},
$$

with

$$
X_{ \pm}^{(j)}=F_{3}^{(j)} \pm \sqrt{\left(F_{5}^{(j)}\right)^{2}+\left(F_{9}^{(j)}\right)^{2}+\left(F_{12}^{(j)}\right)^{2}} .
$$

By multiplying both sides of Eq. (A8) by $\mathcal{X}$, one can arrive at a set of recursive linear algebraic equations for functions $F_{i}^{(j)}$ :

$$
\begin{aligned}
& F_{3}^{j+1}=f_{12} F_{12}^{j}+f_{3} F_{3}^{j}+f_{5} F_{5}^{j}+f_{9} F_{9}^{j}, \\
& F_{9}^{j+1}=-i f_{12} F_{5}^{j}+i f_{5} F_{12}^{j}+f_{3} F_{9}^{j}+f_{9} F_{3}^{j}, \\
& F_{5}^{j+1}=i f_{12} F_{9}^{j}-i f_{9} F_{12}^{j}+f_{3} F_{5}^{j}+f_{5} F_{3}^{j}, \\
& F_{12}^{j+1}=f_{12} F_{3}^{j}+f_{3} F_{12}^{j}+i\left(f_{9} F_{5}^{j}-f_{5} F_{9}^{j}\right),
\end{aligned}
$$

with solutions

$$
\begin{aligned}
& F_{3}^{j}=\frac{1}{2}\left(x_{+}^{j}+x_{-}^{j}\right), \quad F_{9}^{j}=\frac{f_{9}\left(x_{+}^{j}-x_{-}^{j}\right)}{x-y}, \\
& F_{5}^{j}=\frac{f_{5}\left(x_{+}^{j}-x_{-}^{j}\right)}{x-y}, \quad F_{12}^{j}=\frac{f_{12}\left(x_{+}^{j}-x_{-}^{j}\right)}{x-y},
\end{aligned}
$$

which when substituted into Eq. (A9) yield again Eq. (A7).
We will now use this result to calculate the eigenvalues of $\mathcal{X}=\rho^{\mathrm{p} T r}$ as

$$
\begin{aligned}
& \operatorname{det} {[\mathcal{X}-I \lambda] } \\
&=\operatorname{det}[-\lambda I] \exp \left[\sum_{j=1}^{\infty}-\frac{\operatorname{Tr}\left(\mathcal{X}^{j}\right)}{j \lambda^{j}}\right] \\
&=\operatorname{det}[-\lambda I] \exp \left[-\sum_{j=1}^{\infty} \frac{(1-x)^{j}(1-y)^{j}}{j \lambda^{j}\left(1-x^{j}\right)\left(1-y^{j}\right)}\right] .
\end{aligned}
$$

Let us define

$$
\sigma=\frac{\lambda}{\mathcal{N}}=\frac{\lambda}{\left(1-x_{+}\right)\left(1-x_{-}\right)},
$$

then this in combination with

$$
\frac{1}{1-r}=\sum_{i=0}^{\infty} r^{i}
$$

gives us

$$
\begin{aligned}
\operatorname{det}[\mathcal{X}-I \lambda] & =\operatorname{det}[-\lambda I] \exp \left(-\sum_{j=1}^{\infty} \frac{1}{j \sigma^{j}} \sum_{p=0}^{\infty} x_{+}^{p j} \sum_{q=0}^{\infty} x_{-}^{j q}\right) \\
& =\operatorname{det}[-\lambda I] \exp \left[\sum_{p, q=0}^{\infty} \sum_{j=1}^{\infty}-\frac{1}{j}\left(\frac{x_{+}^{p} x_{-}^{q}}{\sigma}\right)^{j}\right] \\
& =\operatorname{det}[-\lambda I] \exp \left[\sum_{p, q=0}^{\infty} \ln \left(1-\frac{x_{+}^{p} x_{-}^{q}}{\sigma}\right)\right] \\
& =\operatorname{det}[-\lambda I] \prod_{p, q=0}^{\infty}\left(1-\frac{x_{+}^{p} x_{-}^{q} \mathcal{N}}{\lambda}\right),
\end{aligned}
$$

so that when the above is equal to zero, it is easy to see that all of the eigenvalues $\lambda_{i}$ are of the form $x_{+}^{p} x_{-}^{q} \mathcal{N}$. Since $x_{+}>0$ and $x_{-}<0$ if $\sqrt{f_{5}^{2}+f_{9}^{2}+f_{12}^{2}}>f_{3}$, then the only negative eigenvalues will be present for odd powers of $x_{-}$and any power of $x_{+}$. Thus, negativity takes the form given by Eq. (5).

\section{The smallest nontrivial problem, the largest with analytically} obtainable transient solutions

The master equation (1) can be solved exactly for an arbitrary initial condition under the parameter reduction 5, i.e., $\kappa_{1}=\kappa_{2}=\kappa$ and $n_{\mathrm{th}, 1}=n_{\mathrm{th}, 2}=n_{\mathrm{th}}$, using the normal ordering solution ansatz

$$
\begin{aligned}
\rho(t)= & e^{f_{0}(t)} e^{f_{3}(t) H_{3}} e^{f_{5}(t) H_{5}} e^{f_{1}(t) H_{1}} e^{f_{6}(t) H_{6}} \\
& \times e^{f_{2}(t) H_{2}} e^{f_{4}(t) H_{4}} \rho(0)
\end{aligned}
$$


and the Wei-Norman method [7,8], we obtain equations

$$
\begin{aligned}
\dot{f}_{1}= & \frac{1}{2}\left\{\kappa\left[2\left(n_{\mathrm{th}}+1\right) f_{3}-2 n_{\mathrm{th}}-1\right]-\frac{1}{2}|\xi| f_{5}\right\}, \\
\dot{f}_{2}= & \frac{1}{2} e^{2 f_{1}}\left[2 \kappa\left(n_{\mathrm{th}}+1\right) \cosh f_{6}-\frac{1}{2}|\xi| \sinh f_{6}\right], \\
\dot{f}_{3}= & -\frac{1}{2}|\xi| f_{3} f_{5}-\kappa\left(2 n_{\mathrm{th}}+1\right) f_{3}+\kappa\left(n_{\mathrm{th}}+1\right) f_{3}^{2} \\
& +\kappa\left[\left(n_{\mathrm{th}}+1\right) f_{5}^{2}+n_{\mathrm{th}}\right], \\
\dot{f}_{4}= & \frac{1}{2} e^{2 f_{1}}\left[\frac{1}{2}|\xi| \cosh f_{6}-2 \kappa\left(n_{\mathrm{th}}+1\right) \sinh f_{6}\right], \\
\dot{f}_{5}= & \kappa f_{5}\left[2\left(n_{\mathrm{th}}+1\right) f_{3}-2 n_{\mathrm{th}}-1\right]-\frac{1}{4}|\xi|\left(f_{3}^{2}+f_{5}^{2}-1\right), \\
\dot{f}_{6}= & 2 \kappa\left(n_{\mathrm{th}}+1\right) f_{5}-\frac{1}{2}|\xi| f_{3},
\end{aligned}
$$

and the last one being $\dot{f}_{0}(t)=\kappa$. These equations linearly decompose into two sets of equations for functions $\left\{p_{+}, q_{+}, r_{+}\right\}$ and $\left\{p_{-}, q_{-}, r_{-}\right\}$such that

$$
\begin{array}{ll}
f_{1}(t)=\frac{1}{4}\left[p_{-}(t)+p_{+}(t)\right], & f_{4}(t)=\frac{1}{2}\left[q_{+}(t)-q_{-}(t)\right], \\
f_{2}(t)=\frac{1}{2}\left[q_{-}(t)+q_{+}(t)\right], & f_{5}(t)=\frac{1}{2}\left[r_{-}(t)-r_{+}(t)\right], \\
f_{3}(t)=\frac{1}{2}\left[r_{-}(t)+r_{+}(t)\right], & f_{6}(t)=\frac{1}{2}\left[p_{-}(t)-p_{+}(t)\right],
\end{array}
$$

where

$$
\begin{aligned}
& p_{ \pm}(t)=2 \ln \left\{\frac{2 e^{\frac{1}{2} t(\kappa \pm|\xi| / 2)}(\kappa \pm|\xi| / 2)}{-2 n_{\mathrm{th}} \kappa+e^{t(\kappa \pm|\xi| / 2)}\left[2\left(n_{\mathrm{th}}+1\right) \kappa \pm|\xi| / 2 \pm|\xi|\right]}\right\} \\
& q_{ \pm}(t)=\frac{\left(1-e^{t(\kappa \pm|\xi| / 2)}\right)\left[2\left(n_{\mathrm{th}}+1\right) \kappa \pm|\xi| / 2\right]}{\left(1-e^{t(\kappa \pm|\xi| / 2)}\right)\left[2\left(n_{\mathrm{th}}+1\right) \kappa \pm|\xi| / 2\right]-2(\kappa \pm|\xi| / 2)} \\
& r_{ \pm}(t)=\frac{\left(1-e^{t(\kappa \pm|\xi| / 2)}\right)\left( \pm|\xi| / 2-2 n_{\mathrm{th}} \kappa\right)}{-2 n_{\mathrm{th}} \kappa+e^{t(\kappa \pm|\xi| / 2)}\left[2\left(n_{\mathrm{th}}+1\right) \kappa \pm|\xi| / 2\right] \pm|\xi| / 2}
\end{aligned}
$$

\section{Details of the initial thermal-state computation}

If the system is initially in the thermal state $\rho(0)=(1-$ $\left.e^{-\beta \hbar \omega}\right) \exp \left(-\beta \hbar \omega \hat{a}^{\dagger} \hat{a}\right)=\left(1-e^{-\beta \hbar \omega}\right) \exp \left(e^{-\beta \hbar \omega} H_{3}\right)|0\rangle\langle 0| \equiv$ $(1-\tau) e^{\tau H_{3}}|0\rangle\langle 0|$, then the evolution takes the form

$$
\begin{aligned}
\rho= & (1-\tau)^{2} e^{f_{0}(t)} e^{f_{3}(t) H_{3}} e^{f_{5}(t) H_{5}} e^{f_{1}(t) H_{1}} e^{f_{6}(t) H_{6}} \\
& \times e^{f_{2}(t) H_{2}} e^{f_{4}(t) H_{4}} e^{\tau H_{3}}|0\rangle\langle 0|
\end{aligned}
$$

This can be rewritten again in the form involving only exponents of $H_{3}$ and $\mathscr{H}_{\theta, 5}$, by means of sandwiching the last exponent in the following manner:

$$
\begin{aligned}
\rho= & (1-\tau)^{2} e^{f_{0}} e^{f_{3} H_{3}} e^{f_{5} H_{5}} e^{f_{1} H_{1}} e^{f_{6} H_{6}} e^{f_{2} H_{2}} e^{f_{4} H_{4}} e^{\tau H_{3}} \\
& \times e^{-f_{4} H_{4}} e^{-f_{2} H_{2}} e^{-f_{6} H_{6}} e^{-f_{1} H_{1}} e^{f_{1} H_{1}} e^{f_{6} H_{6}} e^{f_{2} H_{2}} e^{f_{4} H_{4}}|0\rangle\langle 0| \\
= & (1-\tau)^{2} e^{f_{0}+\frac{1}{2} f_{1}} e^{f_{3} H_{3}} e^{f_{5} H_{5}} e^{f_{1} H_{1}} e^{f_{6} H_{6}} e^{f_{2} H_{2}} e^{f_{4} H_{4}} e^{\tau H_{3}} \\
& \times e^{-f_{4} H_{4}} e^{-f_{2} H_{2}} e^{-f_{6} H_{6}} e^{-f_{1} H_{1}}|0\rangle\langle 0|,
\end{aligned}
$$

which can be brought back to an easier form by realizing that

$$
\begin{aligned}
e^{f_{1} H_{1}} & e^{f_{6} H_{6}} e^{f_{2} H_{2}} e^{f_{4} H_{4}} H_{3} e^{-f_{4} H_{4}} e^{-f_{2} H_{2}} e^{-f_{6} H_{6}} e^{-f_{1} H_{1}} \\
= & \sum_{i=1}^{6} A_{i} H_{i} \equiv \mathcal{J}
\end{aligned}
$$

with

$$
\begin{aligned}
& A_{1}=2 f_{2}, \quad A_{3}=e^{f_{1}} \cosh f_{6}, \\
& A_{6}=2 f_{4}, \quad A_{5}=e^{f_{1}} \sinh f_{6},
\end{aligned}
$$

and

$$
A_{2,4}=\frac{1}{2}\left[e^{-\left(2 f_{1}+f_{6}\right)}\left(f_{2}+f_{4}\right)^{2} \pm e^{-\left(2 f_{1}-f_{6}\right)}\left(f_{2}-f_{4}\right)^{2}\right]
$$

and that $e^{A} e^{\tau B} e^{-B}=e^{\tau e^{A} B e^{-A}}$ gives

$$
\begin{aligned}
& =e^{f_{1} H_{1}} e^{f_{6} H_{6}} e^{f_{2} H_{2}} e^{f_{4} H_{4}} e^{\tau H_{3}} e^{-f_{4} H_{4}} e^{-f_{2} H_{2}} e^{-f_{6} H_{6}} e^{-f_{1} H_{1}} \\
& =\exp \left[\tau \sum_{i=1}^{6} A_{i} H_{i}\right]=e^{\tau \mathcal{J}}
\end{aligned}
$$

and we set out to find $\mathcal{F}_{i}$ such that

$$
e^{\tau \mathcal{J}}=e^{\mathcal{F}_{3} H_{3}} e^{\mathcal{F}_{5} H_{5}} e^{\mathcal{F}_{1} H_{1}} e^{\mathcal{F}_{6} H_{6}} e^{\mathcal{F}_{2} H_{2}} e^{\mathcal{F}_{4} H_{4}}
$$

is another normal ordering decomposition ansatz of an operator exponent. This time, however, it is not a decomposition based on time evolution, but rather the initial condition parameter $\tau$ is acting like an artificial evolution operator which ranges from $0\left(k_{b} T \ll \hbar \omega\right)$ to $1\left(k_{b} T \gg \hbar \omega\right)$. We derive a set of differential equations for functions $\mathcal{F}_{i}$ (the Wei-Norman method) based on

$$
\partial_{\tau} e^{\tau \mathcal{J}}=\mathcal{J} e^{\mathcal{F}_{3} H_{3}} e^{\mathcal{F}_{5} H_{5}} e^{\mathcal{F}_{1} H_{1}} e^{\mathcal{F}_{6} H_{6}} e^{\mathcal{F}_{2} H_{2}} e^{\mathcal{F}_{4} H_{4}},
$$

with the solutions

$$
\begin{aligned}
& \mathcal{P}_{ \pm}=2 \ln \left(1-\tau q_{ \pm}\right), \quad \mathcal{Q}_{ \pm}=\frac{\tau e^{-p_{ \pm}} q_{ \pm}^{2}}{1-\tau q_{ \pm}}, \\
& \mathcal{R}_{ \pm}=\frac{\tau e^{p_{ \pm}}}{1-\tau q_{ \pm}}
\end{aligned}
$$

with

$$
\begin{array}{ll}
\mathcal{F}_{1}(t)=\frac{1}{4}\left[\mathcal{P}_{-}(t)+\mathcal{P}_{+}(t)\right], & \mathcal{F}_{4}(t)=\frac{1}{2}\left[\mathcal{Q}_{+}(t)-\mathcal{Q}_{-}(t)\right], \\
\mathcal{F}_{2}(t)=\frac{1}{2}\left[\mathcal{Q}_{-}(t)+\mathcal{Q}_{+}(t)\right], & \mathcal{F}_{5}(t)=\frac{1}{2}\left[\mathcal{R}_{-}(t)-\mathcal{R}_{+}(t)\right], \\
\mathcal{F}_{3}(t)=\frac{1}{2}\left[\mathcal{R}_{-}(t)+\mathcal{R}_{+}(t)\right], & \mathcal{F}_{6}(t)=\frac{1}{2}\left[\mathcal{P}_{-}(t)-\mathcal{P}_{+}(t)\right],
\end{array}
$$


and then the density operator reads as

$$
\begin{aligned}
\rho= & (1-\tau)^{2} e^{f_{0}+\frac{1}{2} f_{1}} e^{f_{3} H_{3}} e^{f_{5} H_{5}} e^{\mathcal{F}_{3} H_{3}} e^{\mathcal{F}_{5} H_{5}} e^{\mathcal{F}_{1} H_{1}} e^{\mathcal{F}_{6} H_{6}} \\
& \times e^{\mathcal{F}_{2} H_{2}} e^{\mathcal{F}_{4} H_{4}}|0\rangle\langle 0|,
\end{aligned}
$$

which upon the action of the annihilation operators on the vacuum state yields

$$
\rho=\left\{\left[1-g_{3}(t)\right]^{2}-g_{5}(t)^{2}\right\}^{1 / 2} e^{g_{3}(t) H_{3}} e^{g_{5} H_{5}}|0\rangle\langle 0|,
$$

where $g_{i}(t)=f_{i}(t)+\mathcal{F}_{i}(t)$.
It is important to note that only functions $\mathcal{F}_{i}$ carry the information about the initial thermal state stored in the variable $\tau$, and that in the final result only $\mathcal{F}_{3}$ and $\mathcal{F}_{5}$ remain relevant. What is very interesting is that these two functions in the steady state vanish, i.e., $\lim _{t \rightarrow \infty} \mathcal{F}_{3}=0=\lim _{t \rightarrow \infty} \mathcal{F}_{5}$. This means that any impact of this initial-state parameter $\tau$ is completely irrelevant to the steady-state entanglement of the system on both sides of the parameter regimes' boundary $|\xi|=2 \kappa$.
[1] M. O. Scully and M. S. Zubairy, Quantum Optics (Cambridge University Press, Cambridge, UK, 1997).

[2] M. Aspelmeyer, S. Gröblacher, K. Hammerer, and N. Kiesel, J. Opt. Soc. Am. B 27, A189 (2010).

[3] M. Aspelmeyer, T. J. Kippenberg, and F. Marquardt, Rev. Mod. Phys. 86, 1391 (2014).

[4] G. Labadze, M. Dukalski, and Ya. M. Blanter, arXiv:1308.4521.

[5] G. Lindblad, Commun. Math. Phys. 48, 119 (1976).

[6] A. Kossakowski, Rep. Math. Phys. 3, 247 (1972).

[7] J. Wei and E. Norman, J. Math. Phys. 4, 575 (1963).

[8] J. Wei and E. Norman, Proc. Am. Math. Soc. 15, 327 (1964).

[9] M. Ban, J. Math. Phys. 33, 3213 (1992).

[10] C. C. Gerry, Phys. Rev. A 31, 2721 (1985).

[11] C. C. Gerry, Phys. Rev. A 35, 2146 (1987).

[12] G. Dattoli, S. Solimeno, and A. Torre, Phys. Rev. A 34, 2646 (1986).

[13] G. Dattoli, P. Di Lazzaro, and A. Torre, Phys. Rev. A 35, 1582 (1987).
[14] G. Dattoli, M. Richetta, and A. Torre, Phys. Rev. A 37, 2007 (1988).

[15] J. Twamley, Phys. Rev. A 48, 2627 (1993).

[16] J. M. Cerveró and J. D. Lejarreta, J. Phys. A: Math. Gen. 29, 7545 (1996).

[17] M. Horodecki, P. Horodecki, and R. Horodecki, Phys. Lett. A 223, 1 (1996).

[18] G. Vidal and R. F. Werner, Phys. Rev. A 65, 032314 (2002).

[19] R. Horodecki, P. Horodecki, M. Horodecki, and K. Horodecki, Rev. Mod. Phys. 81, 865 (2009).

[20] Lu-Ming Duan, G. Giedke, J. I. Cirac, and P. Zoller, Phys. Rev. Lett. 84, 2722 (2000).

[21] R. Simon, Phys. Rev. Lett. 84, 2726 (2000).

[22] W. K. Wootters, Phys. Rev. Lett. 80, 2245 (1998).

[23] V. Coffman, J. Kundu, and W. K. Wootters, Phys. Rev. A 61, 052306 (2000).

[24] S. M. Barnett and P. L. Knight, J. Opt. Soc. Am. B 2, 467 (1985).

[25] F. Salmistraro and R. Rosso, J. Math. Phys. 34, 3964 (1993). 